IN BRIEF

MICROSCOPY

\section{Mid-IR optoacoustic} microscopy

Pleitez, M. A. et al. Nat. Biotechnol. https://doi. org/10.1038/s41587-019-0359-9 (2019).

Vibrational imaging methods like Raman and infrared microscopy can yield information-rich images of unlabeled cells based on chemical differences among biomolecules. Compared to Raman, mid-infrared spectroscopy and microscopy benefit from biomolecules having high absorbance cross sections, but are limited in biological applications by the absorption from water in these wavelengths, which weakens the measured signal. Pleitez et al. have developed mid-infrared optoacoustic microscopy (MiROM), which combines the benefits of mid-IR excitation and optoacoustic detection. In MiROM, ultrasound waves generated by mid-IR absorption, which are much less attenuated than photons, are measured for improved signal-to-noise and contrast-to-noise imaging. The researchers demonstrate that their approach could be used for diffraction-limited resolution imaging of proteins, carbohydrates and lipids in adipocytes and pancreatic tissue.

https://doi.org/10.1038/s41592-020-0783-2

\section{GENOMICS \\ High-throughput screening with transcriptomics \\ Srivatsan, S. R. et al. Science 367, 45-51 (2020).}

High-throughput screens have been widely used in drug discovery. Yet the typical phenotypic readout, cell viability, often fails to quantify the small changes in cell state that may be attributable to gene expression, epigenetic state or other factors. Srivatsan et al. incorporate 'nuclear hashing' into a single-cell combinatorial indexing (sci-RNA-seq) workflow. The resulting 'sci-Plex' workflow offers a cost-effective highthroughput screen approach for quantifying cellular responses to chemical compounds at single-cell resolution. To this end, the perturbed cells are lysed in-well and subjected to nuclear hashing, meaning their nuclei are labeled with well-specific polyadenylated oligonucleotides. The polyadenylated hashing oligos allow combinatorial indexing together with endogenous mRNAs so that both hash oligos and endogenous mRNA can be recovered. The sci-Plex screening enables profiling thousands of perturbations in a single experiment, and it uncovered dose-dependent responses of a cancer cells line to 188 chemical compounds. $L T$
CHEMICAL BIOLOGY

Biotin 'painting' measures
disorder
Minde, D.-P. et al. Commun. Biol. 3, 38 (2020).

Proteins implicated in diseases such as neurodegeneration, cancer and heart-related disorders are often rich in intrinsically disordered regions (IDRs); however, these proteins remain challenging to study. In particular, the in vivo malleability of these disordered regions, which allows them to adopt different structural forms under different cellular states, has barely been characterized. Minde et al. show through proximity proteomics studies that biotin proximity tagging occurs more favorably in IDRs than in the folded regions of a protein. The researchers further evaluated the time dependence of biotinylation in Escherichia coli ribosomes, in a technique they call 'biotin painting. Biotin labeling was performed for different lengths of time and quantified, providing an estimate of the time course of the structural plasticity of these molecules ranging from unfolded and exposed regions to structured and shielded regions. The assay could be used to obtain in vivo dynamics of intrinsically disordered proteins.

https://doi.org/10.1038/s41592-020-0784-1

\section{NEUROSCIENCE}

\section{A detailed marmoset brain atlas}

Liu, C. et al. Nat. Neurosci. 23, 271-280 (2020).

A detailed description of white matter pathways is paramount for studying connectivity in healthy and diseased primate brains, yet high-resolution atlases of white matter have so far not been available. Liu et al. have built such an atlas for the marmoset. With its small size, the ex vivo marmoset brain could be imaged using high-field MRI and strong gradients. Through optimizing the MRI hardware as well as the scanning protocol and sample preparation, the researchers obtained diffusion-weighted MRI datasets with up to 64-micrometer isotropic resolution, which is unprecedented for primate brains. Owing to the high resolution, the researchers could visualize fine details of fiber pathways that had not been accessible before. Furthermore, the researchers integrated their white matter atlas with a previously acquired atlas of gray matter, as well as with a neuronal tracing database to further increase the value of the atlas. The atlas can be accessed at https://marmosetbrainmapping.org.

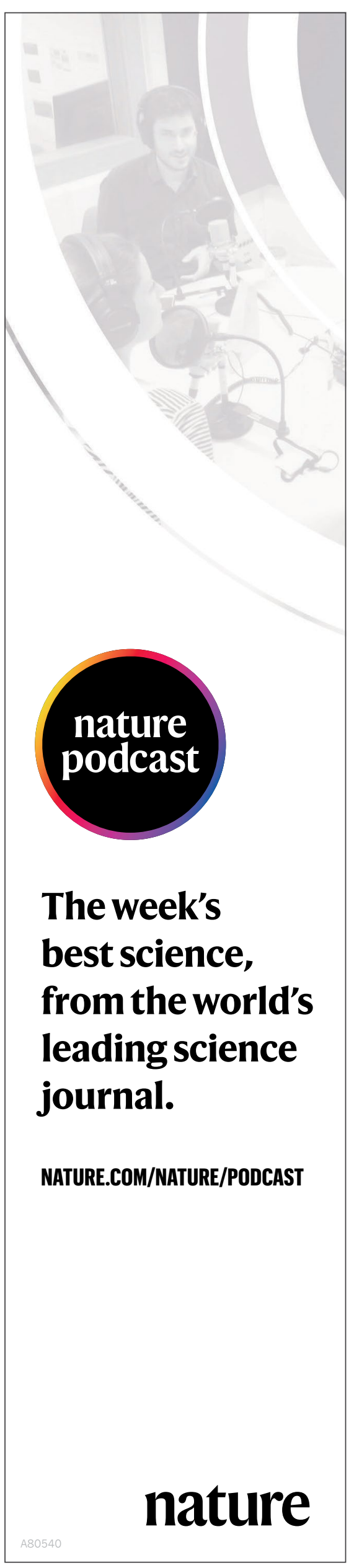

